\title{
Early high antibody titre convalescent plasma for hospitalised COVID-19 patients: DAWn-plasma
}

\author{
Timothy Devos ${ }^{1}$, Quentin Van Thillo $\mathbb{1}^{2}$, Veerle Compernolle ${ }^{3,4}$, Tomé Najdovski ${ }^{5}$, Marta Romano ${ }^{6}$, \\ Nicolas Dauby (i] 7 , Laurent Jadot ${ }^{8}$, Mathias Leys ${ }^{9}$, Evelyne Maillart ${ }^{10}$, Sarah Loof $^{11,12}$, Lucie Seyler $^{13}$, \\ Martial Moonen ${ }^{14}$, Michel Moutschen ${ }^{15}$, Niels Van Regenmortel ${ }^{16}$, Kevin K. Ariën ${ }^{17}$, Cyril Barbezange ${ }^{18}$, \\ Albrecht Betrains ${ }^{19}$, Mutien Garigliany ${ }^{20}$, Matthias M. Engelen ${ }^{21}$, Iwein Gyselinck $\mathbb{B}^{22}$, Piet Maes ${ }^{23}$, \\ Alexander Schauwvlieghe ${ }^{24}$, Laurens Liesenborghs $\mathbb{1}^{25}$, Ann Belmans ${ }^{26,27}$, Peter Verhamme $\mathbb{1}^{21}$ and \\ Geert Meyfroidt ${ }^{28}$ for the DAWn-plasma investigators ${ }^{29}$
}

\begin{abstract}
${ }^{1}$ Dept of Hematology, University Hospitals Leuven and Dept of Microbiology and Immunology, Laboratory of Molecular Immunology (Rega Institute), KU Leuven, Leuven, Belgium. ${ }^{2}$ Center for Cancer Biology, Vlaams Instituut voor Biotechnologie (VIB) and Center for Human Genetics, KU Leuven, Leuven, Belgium. ${ }^{3}$ Belgian Red Cross, Blood Services, Mechelen, Belgium. ${ }^{4}$ Faculty of Medicine and Health Sciences, Ghent University, Ghent, Belgium. ${ }^{5}$ Belgian Red Cross, Service du Sang, Namur, Belgium. ${ }^{6}$ Immune Response Service, Infectious Diseases in Humans Scientific Directorate, Sciensano, Brussels, Belgium. ${ }^{7}$ Dept of Infectious Diseases, CHU Saint-Pierre, School of Public Health, Institute for Medical Immunology, Universite Libre de Bruxelles (ULB), Brussels, Belgium. ${ }^{8}$ Dept of Anesthesiology and Intensive Care Medicine, and Dept of Infectious Diseases, CHC Mont Legia, Liege, Belgium. ${ }^{9}$ Dept of Pulmonary Medicine, AZ Groeninge, Kortrijk, Belgium. ${ }^{10}$ Dept of Infectious Diseases, Brugmann University Hospital, Brussels, Belgium. ${ }^{11}$ Dept of Respiratory Medicine, AZ Maria Middelares Gent, Ghent, Belgium. ${ }^{12}$ Dept of Respiratory Medicine, AZ Sint-Vincentius Deinze, Deinze, Belgium. ${ }^{13}$ Dept of Infectious Diseases and Internal Medicine, UZ Brussel Hospital, Brussels, Belgium. ${ }^{14}$ Dept of Internal Medicine and Infectious Diseases, Centre Hospitalier Regional (CHR), Liege, Belgium. ${ }^{15}$ Infectious Diseases and General Internal Medicine, CHU de Liege, Liege, Belgium. ${ }^{16}$ Dept of Intensive Care Medicine, Ziekenhuis Netwerk Antwerpen Campus Stuivenberg, Antwerp, Belgium. ${ }^{17}$ Virology Unit, Institute of Tropical Medicine Antwerp, Dept of Biomedical Sciences, University of Antwerp, Antwerp, Belgium. ${ }^{18}$ National Influenza Centre, Sciensano, Brussels, Belgium. ${ }^{19}$ Dept of General Internal Medicine, University Hospitals Leuven, Dept of Microbiology, Immunology and Transplantation, KU Leuven, Leuven, Belgium. ${ }^{20}$ Faculty of Veterinary Medicine, Animal Pathology, University of Liege, Liege, Belgium. ${ }^{21}$ Dept of Cardiovascular Sciences, UZ and KU Leuven, Leuven, Belgium. ${ }^{22}$ Laboratory of Respiratory Diseases and Thoracic Surgery (BREATHE), Dept CHROMETA, Respiratory Diseases UZ Leuven, Leuven, Belgium. ${ }^{23}$ Clinical and Epidemiological Virology, Rega Institute for Medical Research, KU Leuven, Leuven, Belgium. ${ }^{24}$ Dept of Haematology, Ghent University Hospital, Ghent, Belgium. ${ }^{25}$ Laboratory of Virology and Chemotherapy, Dept of Microbiology, Immunology and Transplantation, Rega Institute for Medical Research, KU Leuven, Leuven, Belgium. ${ }^{26}$ I-BioStat, KU Leuven, Leuven, Belgium. ${ }^{27}$ University Hasselt, Hasselt, Belgium. ${ }^{28}$ Dept of Intensive Care Medicine, University Hospitals Leuven, Dept of Cellular and Molecular Medicine, Laboratory of Intensive Care Medicine, KU Leuven, Leuven, Belgium. ${ }^{29} \mathrm{~A}$ list of the DAWn-plasma investigators can be found in the Acknowledgements section.
\end{abstract}

Corresponding author: Geert Meyfroidt (geert.meyfroidt@uzleuven.be)

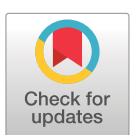

Shareable abstract (@ERSpublications)

Early transfusion of 4 units of high neutralising antibody titre convalescent plasma in hospitalised COVID-19 patients does not reduce mortality or the need for mechanical ventilation https://bit.ly/ 3fiRY2l

Cite this article as: Devos T, Van Thillo Q, Compernolle V, et al. Early high antibody titre convalescent plasma for hospitalised COVID-19 patients: DAWn-plasma. Eur Respir J 2022; 59: 2101724 [DOI: 10.1183/ 13993003.01724-2021].
Copyright $\odot$ The authors 2022

This version is distributed under the terms of the Creative Commons Attribution Non-Commercial Licence 4.0. For commercial reproduction rights and permissions contact permissions@ersnet.org

\section{Abstract}

Background Several randomised clinical trials have studied convalescent plasma for coronavirus disease 2019 (COVID-19) using different protocols, with different severe acute respiratory syndrome coronavirus 2 (SARS-CoV-2) neutralising antibody titres, at different time-points and severities of illness.

Methods In the prospective multicentre DAWn-plasma trial, adult patients hospitalised with COVID-19 were randomised to 4 units of open-label convalescent plasma combined with standard of care (intervention group) or standard of care alone (control group). Plasma from donors with neutralising antibody titres $\left(50 \%\right.$ neutralisation titre $\left.\left(\mathrm{NT}_{50}\right)\right) \geqslant 1 / 320$ was the product of choice for the study.

Results Between 2 May 2020 and 26 January 2021, 320 patients were randomised to convalescent plasma and 163 patients to the control group according to a 2:1 allocation scheme. A median (interquartile range) 
This article has an editorial commentary: https://doi.org/10.1183/ 13993003.02076-2021

Received: 17 June 2021 Accepted: 24 July 2021

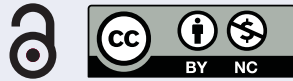

volume of 884 (806-906) $\mathrm{mL}$ ) convalescent plasma was administered and $80.68 \%$ of the units came from donors with neutralising antibody titres $\left(\mathrm{NT}_{50}\right) \geqslant 1 / 320$. Median time from onset of symptoms to randomisation was 7 days. The proportion of patients alive and free of mechanical ventilation on day 15 was not different between both groups (convalescent plasma 83.74\% $(n=267)$ versus control 84.05\% $(n=137)$ ) (OR 0.99, 95\% CI 0.59-1.66; $p=0.9772)$. The intervention did not change the natural course of antibody titres. The number of serious or severe adverse events was similar in both study arms and transfusion-related side-effects were reported in 19 out of 320 patients in the intervention group (5.94\%). Conclusions Transfusion of 4 units of convalescent plasma with high neutralising antibody titres early in hospitalised COVID-19 patients did not result in a significant improvement of clinical status or reduced mortality.

\section{Introduction}

The toll of the coronavirus disease 2019 (COVID-19) pandemic remains high, with 188655968 confirmed cases and 4067517 attributed deaths worldwide as of the 16 July 2021 [1]. Although only a minority of severe acute respiratory syndrome coronavirus 2 (SARS-CoV-2)-infected subjects require hospitalisation, the absolute number of patients presenting with severe or critical illness is large enough to cause near or actual collapse of healthcare systems worldwide [2-4].

The management of hospitalised COVID-19 patients is mainly supportive. So far, three interventions have demonstrated a mortality benefit in hospitalised patients requiring oxygen, primarily targeting the hyperinflammatory phase: dexamethasone [5], tocilizumab [6, 7] and tofacitinib [8]. Therapeutic options in the viral replication phase remain limited. Remdesivir demonstrates little benefit [9] with no impact on mortality [10] and lacks evident antiviral activity in hospitalised patients [11].

The administration of convalescent plasma from donors who have recently recovered from COVID-19 may offer passive immunisation to naïve patients. Randomised clinical trials have studied this therapy in different settings, with different SARS-CoV-2 neutralising antibody titres, at different time-points and severities of illness [12-15]. A recent meta-analysis [16] found no mortality benefit, although heterogeneity between the studies was considered significant. Discrepant findings between different studies might be explained by differences in timing of administration [14, 15], volumes transfused or plasma antibody titres [17].

We hypothesised that giving a high volume of convalescent plasma with high neutralising antibody titres early in hospitalisation for COVID-19 would significantly reduce the proportion of patients who require mechanical ventilation.

\section{Materials and methods}

\section{Study design}

Donated Antibodies Working against nCoV (DAWn-plasma) is a prospective, randomised open-label, multicentre clinical trial to evaluate the efficacy and safety of convalescent plasma added to standard of care in adult patients hospitalised with COVID-19, conducted in 22 Belgian centres, and coordinated by the University Hospitals Leuven, Leuven, Belgium, with public funding by the Belgian Health Care Knowledge Centre (KCE). The trial adhered to the Declaration of Helsinki and Good Clinical Practice principles, had Institutional Review Board approval from the coordinating and participating sites, and was supervised by an independent Data and Safety Monitoring Board. The protocol was publicly registered (ClinicalTrials.gov: NCT04429854) and published [18].

\section{Inclusion and exclusion criteria}

Adult ( $\geqslant 18$ years) hospitalised patients with laboratory or radiologically confirmed COVID-19 were screened for eligibility. In view of the primary end-point, patients receiving mechanical ventilation upon assessment or a therapy restriction code excluding mechanical ventilation and/or endotracheal intubation were excluded. Other exclusion criteria were pregnancy or lactation, a documented previous grade 3 allergic reaction to plasma transfusions and treatment with rituximab or another anti-CD20 monoclonal antibody during the past year. Informed consent was obtained after confirmation of the availability of convalescent plasma prior to randomisation. When written informed consent was not possible due to restrictions for research staff to access the isolation ward, oral consent was documented in the medical file and completed with a signed consent as soon as possible.

\section{Intervention}

Patients were randomised through a computerised system (REDCap version 10.6.13; Vanderbilt University, Nashville, TN, USA) according to a 2:1 allocation scheme stratified by study site using randomly selected 
block sizes of 6 or 9, to open-label convalescent plasma combined with standard of care (intervention group) or standard of care alone (control group). In the intervention group, 2 units of convalescent plasma ( $\sim 200-250 \mathrm{~mL}$ ) were administered within $12 \mathrm{~h}$ after randomisation, with a second administration of 2 units 24-36 h after the first administration. The study protocol did not specify the standard of care therapy.

\section{Selection of donors}

Plasma donations were exclusively obtained from voluntary unpaid donors after informed consent, in accordance with European Union and Belgian legislation for personal data protection. Donors who recovered from a documented SARS-CoV-2 infection (reverse transcriptase (RT)-PCR or radiological confirmation) were recruited in the general population via a web-based interface.

\section{Plasma collection and processing}

Plasma was collected by apheresis using Autopheresis-CTM and Aurora (Fresenius, Willebroek, Belgium) or NexSys (Haemonetics, Signy, Switzerland) equipment. During collection, donor blood was anticoagulated with a citrate solution (sodium citrate dihydrate $4 \%$ ) at a ratio of 1:16. The maximum donated volume allowed per session was $650 \mathrm{~mL}$ (anticoagulant excluded). Methylene blue was used for pathogen reduction of the plasma and plasma was shock-frozen within $18 \mathrm{~h}$ to $-30^{\circ} \mathrm{C}$, over $1 \mathrm{~h}$. Plasma from donors with neutralising antibody titres $\left(50 \%\right.$ neutralisation titre $\left.\left(\mathrm{NT}_{50}\right)\right) \geqslant 1 / 320$ was the product of choice for the study, although titres $\geqslant 1 / 160$ were allowed in case of non-availability. Donor titres were tested monthly.

\section{Neutralising antibody titres}

Anti-SARS-CoV-2 virus neutralisation titres were determined by neutralisation assays, performed in Biosafety Level 3 laboratories in a 96-well plate format, using heat-inactivated plasma or serum samples (30-60 $\mathrm{min}$ at $56^{\circ} \mathrm{C}$ ), as described in the supplementary material. Virus neutralisation titres were reported as $\mathrm{NT}_{50}$.

\section{Viral load measurements}

Nasopharyngeal swabs were placed in a viral transport medium, of which a sample (150 $\mu \mathrm{L})$ was inactivated by adding $600 \mu \mathrm{L}$ RAV1 lysis buffer and subsequent heating for $5 \mathrm{~min}$ at $70^{\circ} \mathrm{C}$. Next, $600 \mu \mathrm{L}$ ethanol was added and total RNA was extracted with the NucleoSpin kit (Macherey-Nagel, Düren, Germany), according to the manufacturer's instructions. RT-quantitative PCR for SARS-CoV-2 was performed on a LightCycler 96 platform (Roche, Pleasanton, CA, USA) with iTaq Universal Probes One-Step RT-qPCR kit (Bio-Rad, Hercules, CA, USA) with N2 primers and probes targeting nucleocapsid16. Standards of known concentrations of SARS-CoV-2 cDNA (IDT, Coralville, IA, USA) were used to extrapolate the total number of viral genome copies per sample.

\section{Study outcomes}

Our primary outcome was the number and proportion of patients alive without mechanical ventilation at day 15. Secondary end-points included clinical status on days 15 and 30, assessed with the World Health Organization 11-point ordinal scale; the time to (whichever comes first) alive hospital discharge or sustained clinical improvement at day 30 (defined as an improvement of $>2$ points versus the highest value of day 0 and 1 and sustained for at least 3 days); all-cause mortality at days 15 and 30; duration of hospital stay; incidence and duration of intensive care unit (ICU) stay and mechanical ventilation; incidence of transfusion-related side-effects and severe adverse events; and quality of life at day 30 (assessed with the EuroQol EQ-5D-5L questionnaire (https://euroqol.org)).

As an exploratory end-point, the correlation between the number of transfused convalescent plasma units from donors with neutralising antibody titres $\left(\mathrm{NT}_{50}\right) \geqslant 1 / 320$ and the primary end-point was analysed. Determinations of viral load in nasal PCR and neutralising antibody titres $\left(\mathrm{NT}_{50}\right)$ in serum samples of patients, both at baseline and day 6, were optional according to protocol and were examined as additional exploratory outcomes when available.

\section{Sample size calculation}

In order to test the superiority hypothesis for a reduction in the proportion of mechanically ventilated patients at day 15 from $16 \%$ in the control group to $7.5 \%$ in the intervention group (a change of $8.5 \%$ ) (with a two-sided type I error rate of 0.050 and a power of 0.8 using a Pearson Chi-squared test for proportion difference), in a 2:1 randomisation scheme, 322 patients needed to be randomised to convalescent plasma and 161 patients to standard of care, yielding a total sample size of 483 patients. 


\section{Statistical analysis}

Statistical analysis was performed in accordance with the International Council for Harmonisation of Technical Requirements for Registration of Pharmaceuticals for Human Use guidelines (version E9; www. ich.org/page/efficacy-guidelines). A detailed description of the analysis is provided in the Statistical Analysis Plan, which was finalised and filed before database lock. A brief summary is provided here.

Analysis sets were finalised during a Blind Review Meeting prior to database lock. The Full Analysis Set (FAS) included all randomised patients, except patients that were confirmed to be SARS-CoV-2-negative and patients who withdrew consent to use any data immediately after randomisation and before treatment administration. The Per Protocol Set (PPS) included all FAS patients in the intervention group that received 4 units of convalescent plasma and all patients in the control group that did not receive any convalescent plasma within 30 days of randomisation.

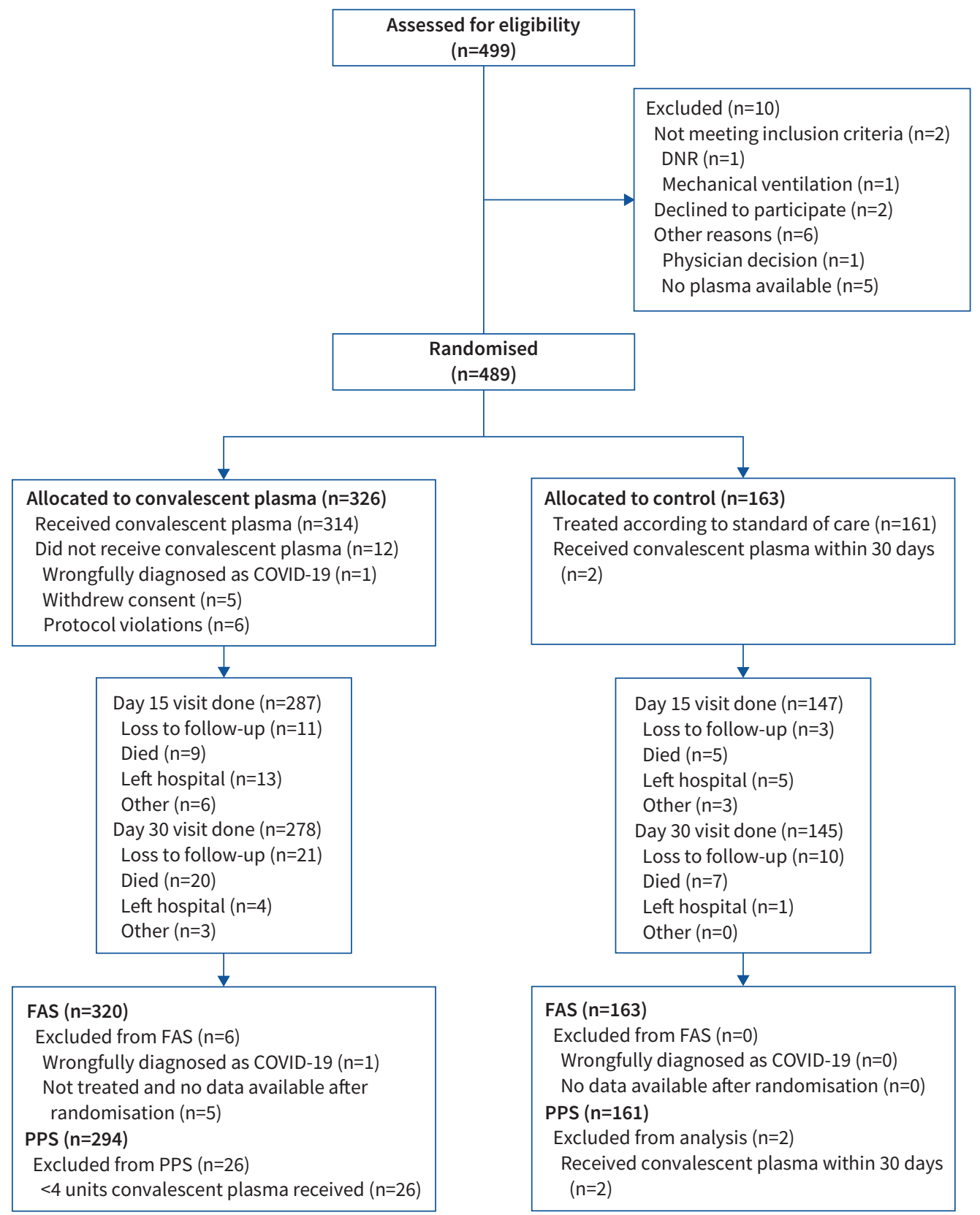

FIGURE 1 CONSORT flow diagram: patient enrolment and treatment assignment. DNR: do not resuscitate; FAS: Full Analysis Set; PPS: Per Protocol Set. 
TABLE 1 Baseline characteristics: Full Analysis Set

\begin{tabular}{|c|c|c|c|c|}
\hline & \multicolumn{2}{|c|}{ Randomised treatment } & \multirow[t]{2}{*}{ Total } & \multirow[t]{2}{*}{$\mathrm{p}$-value } \\
\hline & Plasma+SOC & Soc & & \\
\hline Patients & 320 & 163 & 483 & \\
\hline Age (years) & $62 \pm 14$ & $62 \pm 14$ & $62 \pm 14$ & 0.772 \\
\hline Male & $219 / 320(68.4)$ & $113 / 163(69.3)$ & $332 / 483(68.7)$ & 0.842 \\
\hline Ethnicity & & & & 0.253 \\
\hline Caucasian & $247 / 320(77.2)$ & $135 / 163(82.8)$ & $382 / 483(79.1)$ & \\
\hline North African & $39 / 320(12.2)$ & $20 / 163(12.3)$ & $59 / 483(12.2)$ & \\
\hline Middle Eastern & $16 / 320(5.0)$ & $2 / 163(1.2)$ & $18 / 483(3.7)$ & \\
\hline Black or Sub-Saharan (Africa) & $10 / 320(3.1)$ & $2 / 163(1.2)$ & $12 / 483(2.5)$ & \\
\hline Asian & $5 / 320(1.6)$ & $2 / 163(1.2)$ & $7 / 483(1.5)$ & \\
\hline Latino or Hispanic & $3 / 320(0.9)$ & $2 / 163(1.2)$ & $5 / 483(1.0)$ & \\
\hline $\mathrm{BMI}\left(\mathrm{kg} \cdot \mathrm{m}^{-2}\right)$ & $29 \pm 5(n=264)$ & $30 \pm 6(n=140)$ & $29 \pm 6(n=404)$ & 0.173 \\
\hline History of diabetes mellitus & $98 / 320(30.6)$ & $45 / 163(27.6)$ & $143 / 483(29.6)$ & 0.528 \\
\hline Insulin dependent & $29 / 320(9.1)$ & $17 / 163(10.4)$ & $46 / 483(9.5)$ & 0.628 \\
\hline Oral antidiabetics & $77 / 320(24.1)$ & $23 / 161(14.3)$ & $100 / 481(20.8)$ & 0.013 \\
\hline Smoking status & & & & 0.759 \\
\hline Active & $15 / 316(4.8)$ & $10 / 159(6.3)$ & $25 / 475(5.3)$ & \\
\hline Former & $98 / 316(31.0)$ & $47 / 159(29.6)$ & $145 / 475(30.5)$ & \\
\hline Never & $203 / 316(64.2)$ & $102 / 159(64.2)$ & $305 / 475(64.2)$ & \\
\hline COPD & $29 / 317(9.2)$ & $16 / 160(10.0)$ & $45 / 477(9.4)$ & 0.743 \\
\hline Asthma & $32 / 317(10.1)$ & $16 / 160(10.0)$ & $48 / 477(10.1)$ & 1.000 \\
\hline Heart failure & $26 / 318(8.2)$ & $14 / 159(8.8)$ & $40 / 477(8.4)$ & 0.861 \\
\hline Ischaemic heart disease & $41 / 317(12.9)$ & $26 / 158(16.5)$ & $67 / 475(14.1)$ & 0.328 \\
\hline Chronic kidney disease & $44 / 320(13.8)$ & $20 / 159(12.6)$ & $64 / 479(13.4)$ & 0.777 \\
\hline Kidney disease requiring dialysis & $3 / 318(0.9)$ & $3 / 158(1.9)$ & $6 / 476(1.3)$ & 0.379 \\
\hline Active cancer & $20 / 319(6.3)$ & $9 / 162(5.6)$ & $29 / 481(6.0)$ & 0.841 \\
\hline HIV/AIDS & $3 / 308(1.0)$ & $0 / 157(0.0)$ & $3 / 465(0.7)$ & 0.554 \\
\hline Chronic systemic corticosteroid therapy & $27 / 317(8.5)$ & $17 / 161(10.6)$ & $44 / 478(9.2)$ & 0.504 \\
\hline Other immunosuppressive therapy & $22 / 318(6.9)$ & $17 / 161(10.6)$ & $39 / 479(8.1)$ & 0.215 \\
\hline Highest body temperature $\left({ }^{\circ} \mathrm{C}\right)$ & $37.6 \pm 1.0(n=316)$ & $37.7 \pm 1.0(n=162)$ & $37.7 \pm 1.0(n=478)$ & 0.171 \\
\hline Lowest oxygen saturation when breathing room air (\%) & $91.0(88.0-93.0)(n=272)$ & $91.0(86.0-94.0)(n=127)$ & $91.0(87.0-93.0)(n=399)$ & 0.792 \\
\hline Oxygen therapy & & & & 0.599 \\
\hline No & $36 / 320(11.3)$ & $21 / 163(12.9)$ & $57 / 483(11.8)$ & \\
\hline Yes & $284 / 320(88.8)$ & $142 / 163(87.1)$ & $426 / 483(88.2)$ & \\
\hline Consciousness level & & & & 1.000 \\
\hline Alert & $310 / 320(96.9)$ & $157 / 163(96.3)$ & $467 / 483(96.7)$ & \\
\hline Verbal & $9 / 320(2.8)$ & $5 / 163(3.1)$ & $14 / 483(2.9)$ & \\
\hline Pain & $1 / 320(0.3)$ & $1 / 163(0.6)$ & $2 / 483(0.4)$ & \\
\hline Disease triage at admission & & & & 0.698 \\
\hline Ward & $262 / 320(81.9)$ & $135 / 161(83.9)$ & $397 / 481(82.5)$ & \\
\hline Intensive care unit & $48 / 320(15.0)$ & $23 / 161(14.3)$ & $71 / 481(14.8)$ & \\
\hline Emergency room & $10 / 320(3.1)$ & $3 / 161$ (1.9) & $13 / 481(2.7)$ & \\
\hline
\end{tabular}

Data are presented as $\mathrm{n}$, mean $\pm \mathrm{SD}, \mathrm{n} / \mathrm{N}(\%)$ or median (interquartile range), unless otherwise stated. SOC: standard of care; BMI: body mass index; COPD: chronic obstructive pulmonary disease. Continuous variables were compared using the two-sample t-test. Categorical variables were compared using the Chi-squared test.

Missing clinical status data were accounted for by means of multiple imputation, using a total of 100 imputations [19]. Treatment effects for all end-points were estimated by an appropriate measure and presented with 95\% confidence intervals, and were adjusted for study site and period. The primary end-point was compared using logistic regression to estimate the odds ratio. Pre-specified subgroup analyses were performed for the primary end-point only, considering the following subgroups of interest: duration of symptoms prior to enrolment (according to observed median), age groups (according to observed median), study period, blood group, size and province of study site, primary admission to the ICU, and blood institute that processed the convalescent plasma. All-cause mortality and survival without mechanical ventilation up to 30 days were assessed using a Cox regression to obtain hazard ratios. Incidence rates were estimated using Kaplan-Meier methodology. Time to sustained improvement, incidence and duration of supplemental oxygen, mechanical ventilation, extracorporeal membrane 
TABLE 2 Trial primary and secondary end-points: Full Analysis Set $(n=483)$

\begin{tabular}{|c|c|c|c|c|c|}
\hline & \multirow[t]{2}{*}{ Statistic } & \multicolumn{2}{|c|}{ Estimate $(95 \% \mathrm{Cl})$} & \multirow[t]{2}{*}{ Treatment effect } & \multirow[t]{2}{*}{ Estimate $(95 \% \mathrm{Cl})$} \\
\hline & & Plasma & SOC & & \\
\hline Alive and free of mechanical ventilation at 15 days & $\%$ & $83.7(79.3-87.4)$ & $84.1(77.6-88.9)$ & OR & $0.99(0.59-1.68)$ \\
\hline Alive and free of mechanical ventilation at 30 days & KM (\%) & $82.5(78.1-86.4)$ & $82.2(76.0-87.6)$ & $\mathrm{HR}$ & $0.94(0.60-1.48)$ \\
\hline Sustained improvement or discharge within 30 days & $\mathrm{CIF}(\%)$ & $82.6(77.9-86.3)$ & $84.7(78.1-89.4)$ & Subdistribution HR & $0.98(0.81-1.20)$ \\
\hline Hospital discharge (30 days) & CIF $(\%)$ & $80.5(75.7-84.4)$ & $79.8(72.8-85.2)$ & Subdistribution HR & $1.06(0.87-1.30)$ \\
\hline \multicolumn{6}{|l|}{ All-cause mortality } \\
\hline Day 15 & KM (\%) & $3.1(1.7-5.8)$ & $4.9(2.5-9.6)$ & $\mathrm{HR}$ & $0.61(0.24-1.54)$ \\
\hline Day 30 & KM (\%) & $9.1(6.3-12.9)$ & $8.7(5.3-14.3)$ & $\mathrm{HR}$ & $0.99(0.52-1.88)$ \\
\hline \multicolumn{6}{|l|}{ Supplemental oxygen (30 days) } \\
\hline Incidence & CIF (\%) & $89.5(85.5-92.4)$ & $89.0(83.0-92.9)$ & Subdistribution HR & $1.01(0.93-1.09)$ \\
\hline Life-weaning from supplemental oxygen & CIF (\%) & $80.7(75.6-84.8)$ & $82.3(74.9-87.7)$ & Subdistribution HR & $1.05(0.86-1.29)$ \\
\hline \multicolumn{6}{|l|}{ Mechanical ventilation ( 30 days) } \\
\hline Incidence & CIF (\%) & $15.0(11.3-19.2)$ & $13.5(8.8-19.2)$ & Subdistribution HR & $1.08(0.65-1.80)$ \\
\hline Life-weaning from mechanical ventilation & $\mathrm{CIF}(\%)$ & $58.4(42.1-71.5)$ & $68.2(43.3-83.9)$ & Subdistribution HR & $0.49(0.22-1.08)$ \\
\hline \multicolumn{6}{|l|}{ ICU (30 days) } \\
\hline Admission & $\mathrm{CIF}(\%)$ & $36.0(30.8-41.3)$ & $34.4(27.2-41.7)$ & Subdistribution HR & $1.00(0.74-1.34)$ \\
\hline Life discharge & CIF (\%) & $78.3(69.5-84.8)$ & $82.1(69.0-90.1)$ & Subdistribution HR & $0.95(0.66-1.35)$ \\
\hline \multicolumn{6}{|l|}{ Clinical status } \\
\hline Day 0 & Median (IQR) & $5(5-5)$ & $5(5-5)$ & & \\
\hline Day 15 & Median (IQR) & $2(0-5)$ & $2(0-5)$ & Common OR & $1.09(0.78-1.53)$ \\
\hline Day 30 & Median (IQR) & $2(0-2)$ & $2(0-3)$ & Common OR & $0.95(0.67-1.33)$ \\
\hline \multicolumn{6}{|l|}{ EQ-5D-5L } \\
\hline Baseline & Mean \pm SD & $54 \pm 18$ & $54 \pm 18$ & & \\
\hline Day 30 & Mean \pm SD & $73 \pm 16$ & $72 \pm 17$ & Mean difference & $1.32(-2.24-4.88)$ \\
\hline \multicolumn{6}{|l|}{$\mathrm{NT}_{50}$ values } \\
\hline Day 0 (log 2 -transformed) & Median (IQR) & $3(1-5)$ & $3(1-5)$ & & \\
\hline Day 6 ( $\log _{2}$-transformed) & Median (IQR) & $6(5-6)$ & $6(5-6)$ & Mean difference & $0.08(-0.43-0.58)$ \\
\hline Ratio day $6 /$ day 0 ( $\log _{2}$-transformed) & Median (IQR) & $2(1-3)$ & $2(0-4)$ & Mean difference & $0.03(-0.62-0.68)$ \\
\hline \multicolumn{6}{|c|}{$\begin{array}{l}\text { SOC: standard of care; ICU: intensive care unit; } \mathrm{NT}_{50} \text { : } 50 \% \text { neutralisation titre; } \mathrm{KM} \text { : incidence estimated using Kaplan-Meier methodology; } 95 \% \\
\mathrm{Cl} \text { : } 95 \% \text { confidence interval calculated using log(- } \mathrm{log} \text { ) transformation; CIF: incidence estimated using the cumulative incidence function accounting } \\
\text { for competing risks; IQR: interquartile range; HR: hazard ratio; OR: odds ratio. All estimates of treatment effects were adjusted for study site and } \\
\text { period. Hazard ratios were obtained using a Cox regression including factors for randomised treatment, study period and site. Subdistribution } \\
\text { hazard ratios were obtained using a Fine-Gray regression model (accounting for competing risks) including factors for randomised treatment, study } \\
\text { period and site. Mean differences between treatments were obtained using a general linear model including baseline value as a covariate and } \\
\text { factors for randomised treatment, study period and site. Common odds ratios were obtained using a proportional odds logistic regression analysis } \\
\text { including baseline clinical status as a covariate and factors for randomised treatment, study period and site. }\end{array}$} \\
\hline
\end{tabular}

oxygenation, and ICU were analysed using competing risks methodology, using cumulative incidence functions to estimate event rates and a Fine-Gray regression model to obtain cause-specific hazard ratios. All tests were two-sided and assessed at a significance level of 5\%. No correction was made for multiple secondary end-points. All analyses were performed using SAS version 9.4 for Windows 10 (SAS Institute, Cary, NC, USA).

Results

\section{Patients}

Between 2 May 2020 and 26 January 2021, 499 patients were assessed for eligibility, of whom 489 were randomised to convalescent plasma $(n=326)$ or control $(n=163)$ (figure 1). The FAS consisted of 320 patients in the plasma group and 163 in the control group. Baseline and demographic data are summarised in table 1; both groups were well matched. Concomitant therapy for COVID-19 was similar between both groups (supplementary material). Median (IQR) time from symptom onset to hospital admission was 6 (3-8) days and from admission to randomisation was 1 (1-2) days in both groups. In the convalescent plasma group, the median (IQR) time from randomisation to the first plasma transfusion was 5 (4-7) h and a median (IQR) volume of $884(807-906) \mathrm{mL}$ convalescent plasma was administered. 80.7\% of the administered plasma units (981 out of 1215 units) came from donors with neutralising antibody titres of at least 1/320. Six patients in the plasma group of the FAS never received convalescent plasma; 294 (91.9\%) patients received all 4 units and were included in the PPS. 


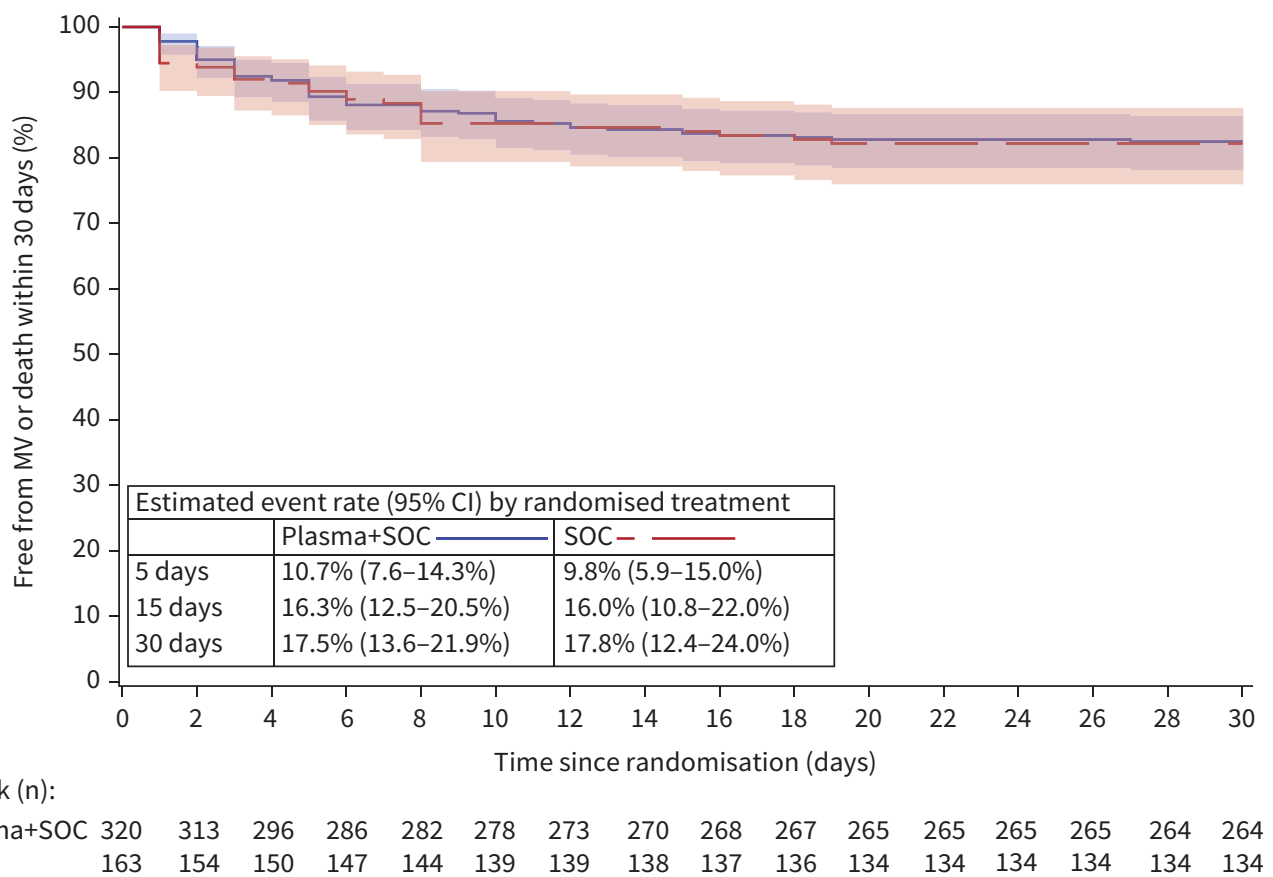

FIGURE 2 Kaplan-Meier curve. Proportion of patients remaining free of mechanical ventilation (MV) or death: Full Analysis Set. SOC: standard of care.

\section{Primary outcome}

The proportion of patients alive and free of mechanical ventilation on day 15 was not different between both groups in the FAS (convalescent plasma 83.7\% $(n=266)$ versus control 84.1\% ( $\mathrm{n}=137)$ ) (OR 0.99, 95\% CI 0.59-1.68; p=0.976) (table 2). Kaplan-Meier curves are depicted in figure 2. Results were similar for the PPS (supplementary material). Pre-specified subgroup analyses for the primary end-point (figure 3) demonstrated a significant interaction with age $(\mathrm{p}=0.023)$.

\section{Secondary outcomes}

Secondary end-points are summarised in table 2. No difference was detected in the proportion of patients alive and free of mechanical ventilation on day 30 (figure 2), or any of the other secondary end-points on day 15 or day 30.

\section{Exploratory end-points}

There was no significant association between the number of units transfused with neutralising antibody titre $\geqslant 1 / 320$ and outcome (figure 4). At baseline, 30\% (33 out of 110) of patients in the plasma group and $26 \%$ (14 out of 53) of patients in the control group already had neutralising antibody serum titres of $\geqslant 1 /$ 320. Titres of neutralising antibodies against SARS-CoV-2 $\left(\mathrm{NT}_{50}\right)$ increased between baseline and day 6 after randomisation, but this increase was not influenced by the intervention (estimated difference in $\log _{2}$-transformed day 6 values between study groups, adjusted for baseline: 0.08 , 95\% CI $-0.43-0.58$; $\mathrm{p}=0.766$ ) (figure 5). A better outcome was correlated with higher neutralising antibody titres at day 0 (OR of good outcome for increase of 1 in $\log _{2}$-transformed $\mathrm{NT}_{50}$ at day $0: 1.45$, 95\% CI 1.11-1.83; $\mathrm{p}=0.005$ ) and day 6 (OR 1.68, 95\% CI 1.30-2.16; $\mathrm{p}<0.001$ ) (figure 6), but not with the magnitude of increase in $\mathrm{NT}_{50}$ between day 0 and day 6. Viral load decreased in a similar manner in both treatment groups (estimated treatment difference of $\log _{10}$-transformed day 6 values, adjusted for baseline: 1.70, 95\% CI $0.40-7.21 ; \mathrm{p}=0.466$ ) (figure 7).

There were no significant interactions between fraction of inhaled oxygen at baseline $(\mathrm{p}=0.0906)$ or time from symptoms to randomisation $(\mathrm{p}=0.9386)$ and randomised treatment in their effect on the primary outcome (supplementary material).

\section{Safety}

Numbers of serious or severe adverse events reported were similar in both study arms: 20.6\% (66 out of 320 ) in the plasma arm and 22.1\% (36 out of 163) in the control group (supplementary material). 


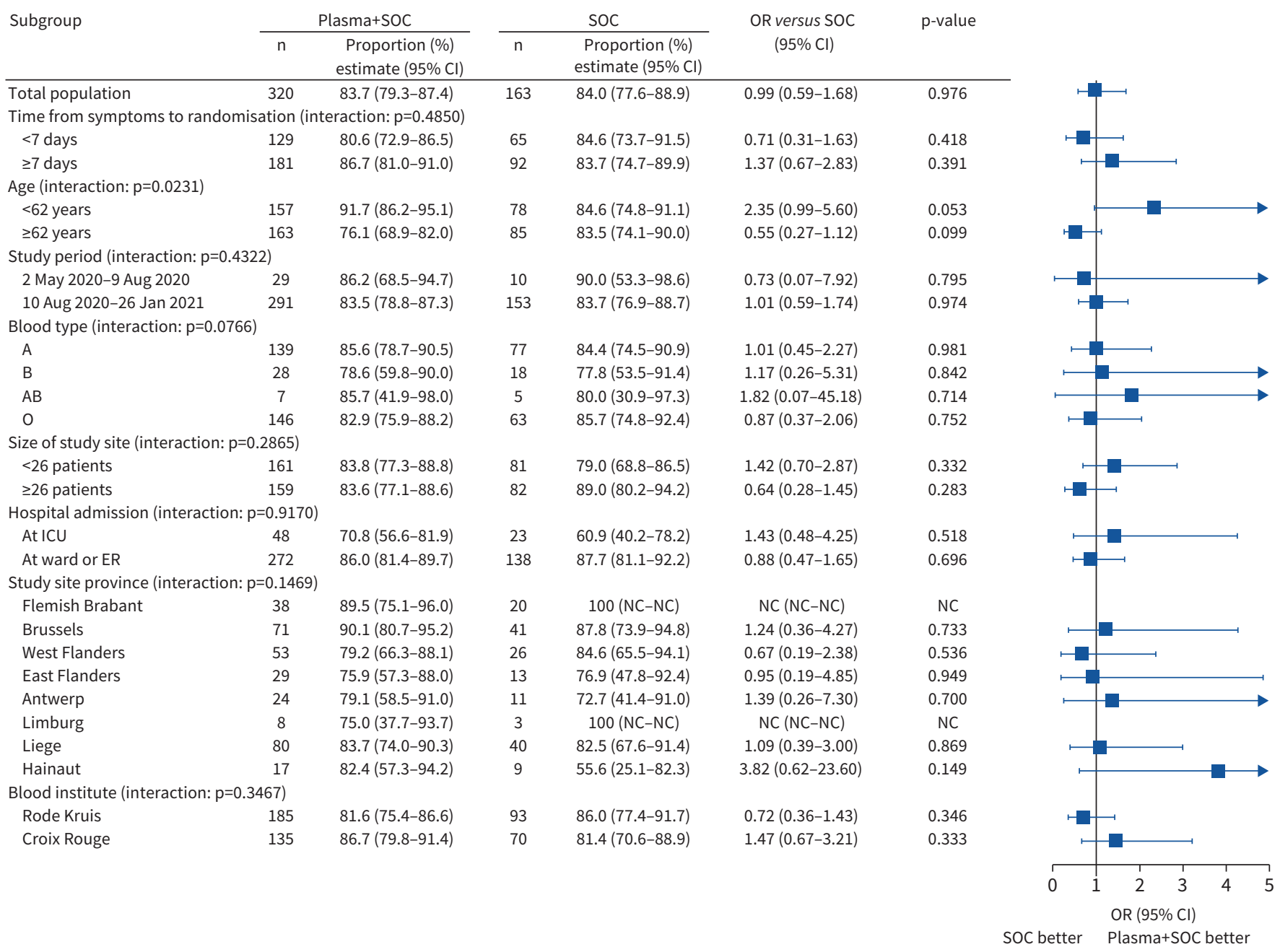

FIGURE 3 Pre-specified subgroup analysis for the primary end-point: Full Analysis Set. The odds ratios were obtained by means of a logistic regression that included factors for treatment, study site, study period, subgroup, and interaction between treatment and subgroup. For blood institute, study site was not included in the model due to problems with fitting the model. SOC: standard of care; ICU: intensive care unit; ER: emergency room; NC: not calculated.

Transfusion-related side-effects were reported in 19 out of 320 patients in the intervention group (5.9\%) (supplementary material).

\section{Discussion}

In the DAWn-plasma study, the administration of high-volume (median total volume $884 \mathrm{~mL}$ ), high-titre convalescent plasma early in hospitalisation for COVID-19 disease did not succeed in reducing the need for mechanical ventilation at day 15 (primary end-point) or have an impact on any of the secondary outcomes, including the need for and the duration of ICU stay, and mortality and quality of life at day 30 . Administration of convalescent plasma was safe as no major adverse events were registered and transfusion reactions were in the expected range of occurrence.

These results are in line with other trials on convalescent plasma for COVID-19, as evident from a recent meta-analysis of published and unpublished trials [16], including the large RECOVERY study [20]. Even while $>80 \%$ of the units came from donors with neutralising antibody titres $\geqslant 1 / 320$ and the volume of plasma transfused was higher than any other published trial, the intervention did not succeed in influencing the natural course of SARS-CoV-2 neutralising antibodies or viral load. The median time between onset of symptoms and randomisation was 7 days, which might have been too late to obtain a meaningful clinical effect. The finding that $28.8 \%$ of patients already showed significantly elevated $(\geqslant 1 / 320)$ serum neutralising antibodies at baseline supports this hypothesis. However, from a pragmatic point of view, the 


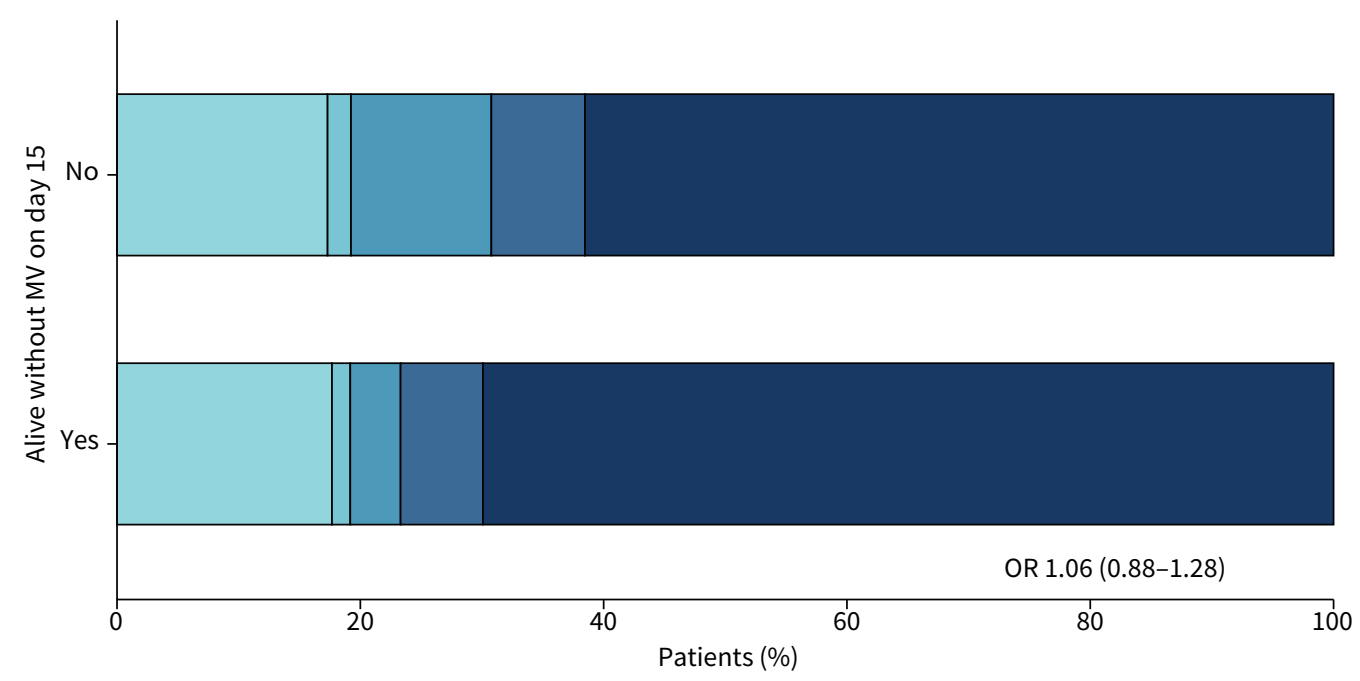

Transfused units with antibody titre $\geq 1 / 320: \quad \square 0 \quad \square 1 \square 2 \square 3 \square 4$

FIGURE 4 Outcome according to antibody titre in donor plasma: Full Analysis Set. Association between the number of plasma units with antibody titre $\geqslant 1 / 320$ given to patients and outcome (alive without mechanical ventilation (MV) on day 15). The odds ratio reflects the change in odds for being alive with no MV on day 15 when the number of units $\geqslant 1 / 320$ increases by 1 .

timing of presentation to the hospital is a clinical reality and the administration of blood products in the pre-hospital phase is not routine clinical practice in Belgium, like in many other countries. Given the short timeframe of 1 day between hospital admission and randomisation, it is unlikely that convalescent plasma could have been administered earlier in the Belgian setting. In addition, since time from symptom onset to randomisation was not a significant interaction term, it seems unlikely that the results would have been different with earlier administration. The overall all-cause mortality in our trial ( $8.8 \%$ at 30 days), both in

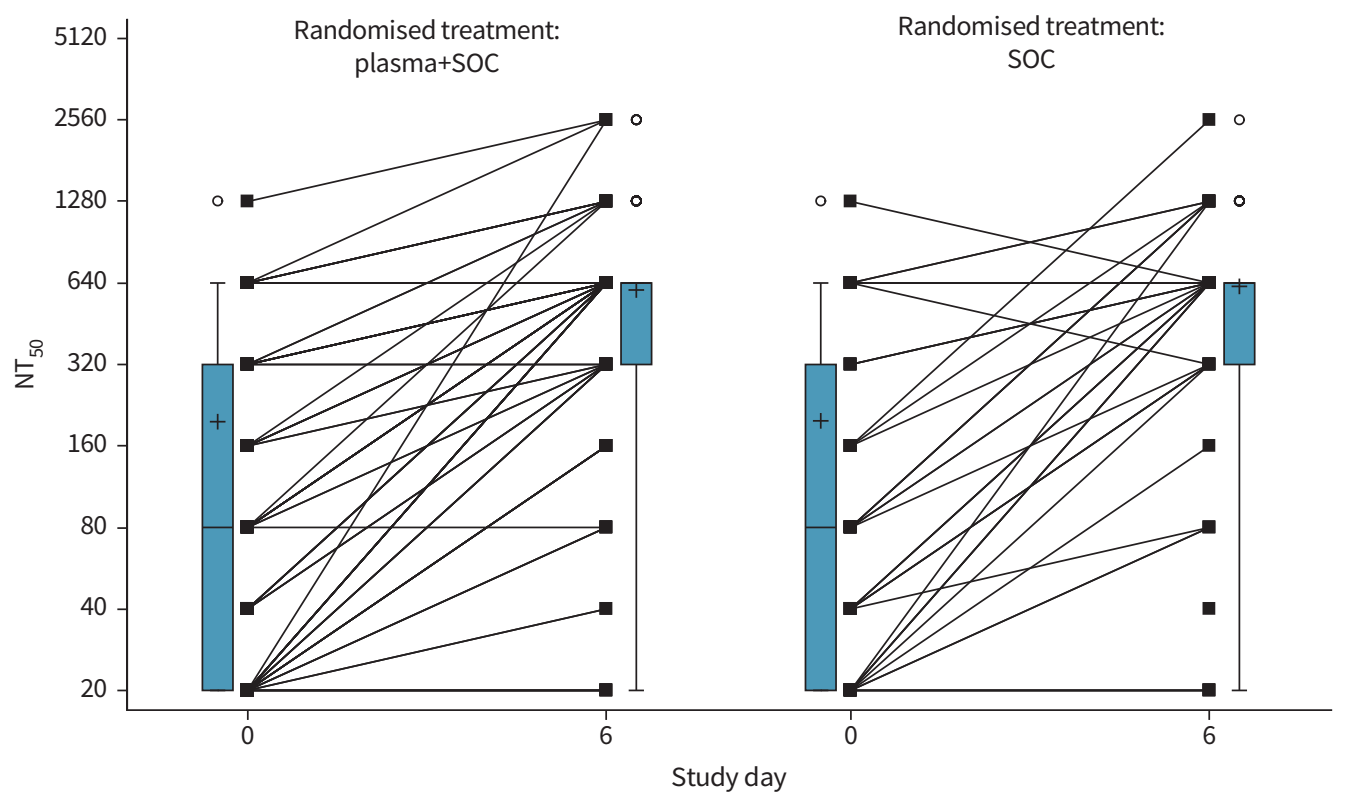

FIGURE 5 Evolution of neutralising antibody titres ( $50 \%$ neutralisation titre $\left.\left(\mathrm{NT}_{50}\right)\right)$ in patient sera. Box plots shows median and interquartile range (IQR). Whiskers are drawn at 1.5 IQR. Mean values are indicated by "+". Circles indicate outlying values. Lines connect individual patient values (squares) between day 0 and 6. SOC: standard of care. 

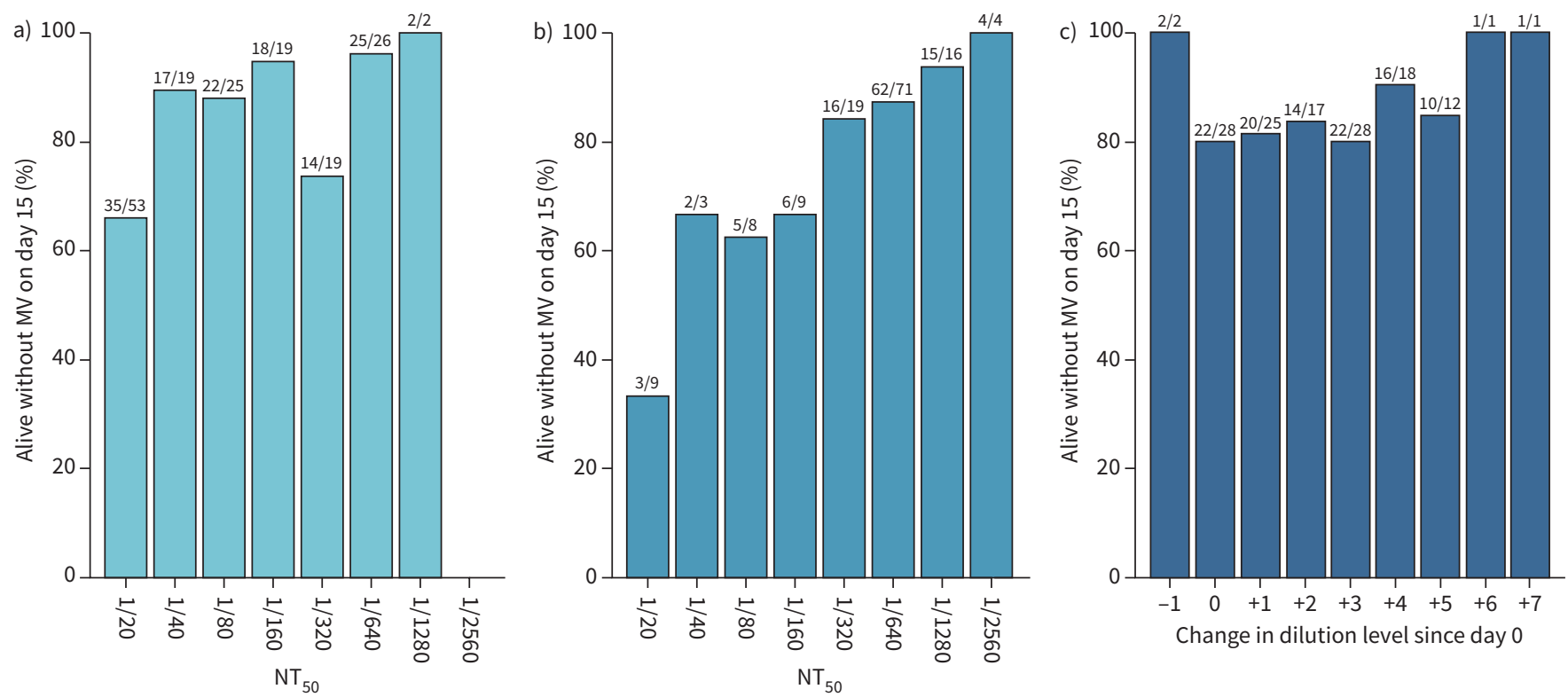

FIGURE 6 Neutralising antibody titre (50\% neutralisation titre $\left.\left(\mathrm{NT}_{50}\right)\right)$ relation to primary outcome. Number and proportion of patients alive without mechanical ventilation (MV) on day 15 according to $\mathrm{NT}_{50}$ titre levels on a) day 0 and b) day 6 , and c) according to the change in titre levels between day 0 and day 6 . Results above the bars are shown as $n / N$, where $n=$ number of patients alive without MV on day 15 in the category and $\mathrm{N}=$ total number of patients in the category.

the plasma and the control group, is relatively low compared with the control group mortality of $12.7 \%$ in previously published peer-reviewed trials [16], $24 \%$ in the RECOVERY trial [20] or $24.6 \%$ in the control group of the O'Donnell et al. [21] trial. As such, our findings might not translate to other settings,

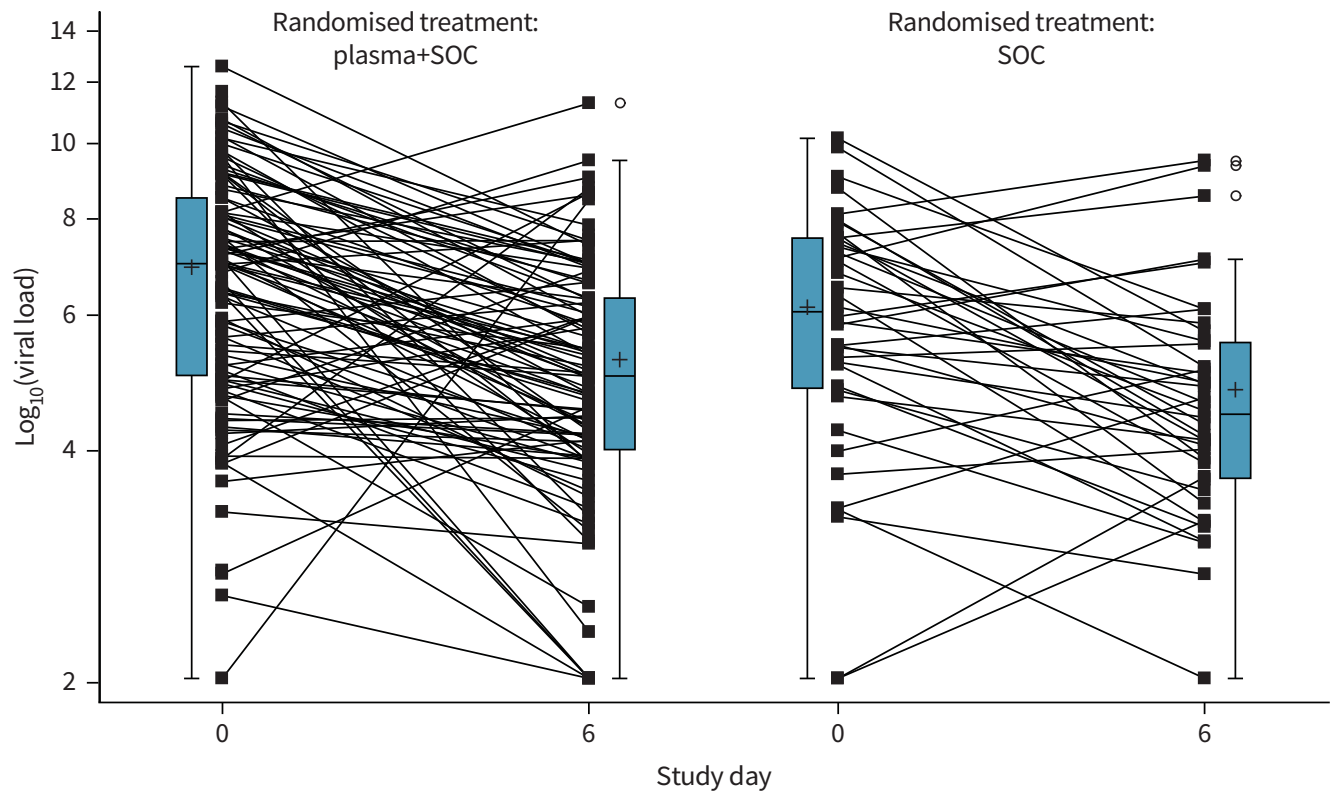

FIGURE 7 Evolution of viral load (baseline versus day 6 since randomisation): Full Analysis Set. Box plots show median and interquartile range (IQR). Whiskers are drawn at 1.5 IQR. Mean values are indicated by "+". Circles indicate outlying values. Below the limit of quantification values were imputed with half of the minimum observed value. $\log _{10}$-transformed viral load values at day 6 were compared between groups using a general linear model with factors for treatment, study site and period, and including baseline value as a covariate. The resulting estimate of the treatment difference was $0.23(95 \% \mathrm{Cl}-0.40-0.86)$. 
potentially representing a different case mix, different hospital systems or a different degree of healthcare system overflow.

In our study, a significant interaction was found between plasma administration and age, whereby plasma administration was associated with improved clinical outcome in younger patients. No such age interaction was observed in a placebo-controlled convalescent plasma trial [13] with exactly the same median age of patients as in our study. In view of the overall lack of benefit of the intervention across several trials, it is debatable whether future studies should focus on the younger population based on this interaction analysis.

Our study has several limitations. The study was designed as an open-label study, where the intervention was not blinded. No placebo treatment was given. Six study patients were excluded post-randomisation because of early withdrawal, all in the intervention group. Only $91 \%$ of patients received the intervention strictly per protocol and almost $20 \%$ of convalescent plasma units did not contain the pre-specified $\geqslant 1 / 320$ neutralising antibody titres.

Patients treated with B-lymphocyte-depleting monoclonal antibodies during the year before admission were excluded from participation in the DAWn-plasma study. As such, the results of our study cannot be extrapolated to these patients, often not clearing the SARS-CoV-2 virus, where convalescent plasma could still be considered [22, 23]. Lastly, the study was largely conducted before the appearance of new variants of SARS-CoV-2 in Belgium.

In summary, transfusion of a high volume of 4 units of convalescent plasma with high neutralising antibody titres early in hospitalised COVID-19 patients could not change the natural course of antibody titres and did not result in a significant improvement of the clinical status, nor did the intervention reduce mortality.

Acknowledgements: The acknowledgements can be found in the supplementary material.

The DAWn-plasma investigators: Georgala Aspasia, Nina Bijnens, Kris Bogaerts, Bernard Bouckaert, Helga Ceunen, Myriam Cleeren, Jan Cools, Kristof Cuppens, Barbara Debaveye, Melanie Delvallee, Paul De Munter, Daniel Desmecht, Elke Govaerts, David Grimaldi, Wim Janssens, Johan Neyts, Jill Pannecoucke, Elisabeth Porcher, Camelia Rossi, Thomas Van Assche, Katleen Vandenberghe, Emmanuel Van der Hauwaert, Steven Vanderschueren, Eric Van Wijngaerden, Geert Verbeke, Clothilde Visee, Robin Vos and Jean Cyr Yombi.

This study is registered at ClinicalTrials.gov with identifier number NCT04429854. DAWn-plasma trial data are also shared and analysed for meta-analysis in several collaborations and consortia (e.g. Compile consortium and others). Agreements in this context do not allow the authors to make data available.

Author contributions: Statistical analysis was done by the principal investigator (G. Meyfroidt) and the study statistician (A. Belmans). The first draft of the manuscript was written by T. Devos and G. Meyfroidt; specific sections were written by the writing committee. All authors have read and approved the final manuscript, and take responsibility for the accuracy and completeness of the data as well as adherence to the protocol. Contributions of individual authors are listed in the supplementary material.

Conflict of interest: All authors report support for the present manuscript from the Belgian Healthcare Knowledge Center (KCE). Q. Van Thillo reports grants from Fonds Wetenschappelijk Onderzoek (FWO)-Vlaanderen Basic Research 2019-2021 outside the submitted work. G. Meyfroidt reports a FWO-Vlaanderen Senior Clinical Researcher Grant outside the submitted work.

Support statement: This work was supported by Fonds Wetenschappelijk Onderzoek (grant: 1843118N) and Belgian Health Care Knowledge Centre (KCE). Funding information for this article has been deposited with the Crossref Funder Registry.

\section{References}

1 World Health Organization. WHO coronavirus (COVID-19) dashboard with vaccination data. 2021. https:// covid19.who.int Date last accessed: 16 July 2021.

2 Grasselli G, Pesenti A, Cecconi M. Critical care utilization for the COVID-19 outbreak in Lombardy, Italy: early experience and forecast during an emergency response. JAMA 2020; 323: 1545-1546.

3 Verity R, Okell LC, Dorigatti I, et al. Estimates of the severity of coronavirus disease 2019: a model-based analysis. Lancet Infect Dis 2020; 20: 669-677. 
4 Taccone FS, Van Goethem N, De Pauw R, et al. The role of organizational characteristics on the outcome of COVID-19 patients admitted to the ICU in Belgium. Lancet Reg Health Eur 2021; 2: 100019.

5 The RECOVERY Collaborative Group. Dexamethasone in hospitalized patients with Covid-19. N Engl J Med 2021; 384: 693-704.

6 The REMA-CAP Investigators. Interleukin-6 receptor antagonists in critically ill patients with Covid-19. N Engl J Med 2021; 84: 1491-1502.

7 Salama C, Han J, Yau L, et al. Tocilizumab in patients hospitalized with Covid-19 pneumonia. N Engl J Med 2021; 384: 20-30.

8 Guimarães PO, Quirk D, Furtado RH, et al. Tofacitinib in patients hospitalized with Covid-19 pneumonia. N Engl J Med 2021; 385: 406-415.

9 Beigel JH, Tomashek KM, Dodd LE, et al. Remdesivir for the treatment of Covid-19 - final report. N Engl J Med 2020; 383: 1813-1826.

10 WHO Solidarity Trial Consortium. Repurposed antiviral drugs for COVID-19 - interim WHO Solidarity trial results. N Engl J Med 2021; 384: 497-511.

11 Goldberg E, Ben Zvi H, Sheena L, et al. A real-life setting evaluation of the effect of remdesivir on viral load in COVID-19 patients admitted to a large tertiary centre in Israel. Clin Microbiol Infect 2021; 27: 917.e1-917.e4.

12 Agarwal A, Mukherjee A, Kumar G, et al. Convalescent plasma in the management of moderate covid-19 in adults in India: open label phase II multicentre randomised controlled trial (PLACID Trial). BMJ 2020; 371: m3939.

13 Simonovich VA, Burgos Pratx LD, Scibona P, et al. A randomized trial of convalescent plasma in Covid-19 severe pneumonia. N Engl J Med 2021; 384: 619-629.

14 Gharbharan A, Jordans C, GeurtsvanKessel C, et al. Effects of potent neutralizing antibodies from convalescent plasma in patients hospitalized for severe SARS-CoV-2 infection. Nat Commun 2020; 12: 3189.

15 Libster R, Pérez Marc G, Wappner D, et al. Early high-titer plasma therapy to prevent severe Covid-19 in older adults. N Engl J Med 2021; 384: 610-618.

16 Janiaud P, Axfors C, Schmitt AM, et al. Association of convalescent plasma treatment with clinical outcomes in patients with COVID-19: a systematic review and meta-analysis. JAMA 2021; 325: 1185-1195.

17 Joyner MJ, Carter RE, Senefeld JW, et al. Convalescent plasma antibody levels and the risk of death from Covid-19. N Engl J Med 2021; 384: 1015-1027.

18 Devos T, Geukens T, Schauwvlieghe A, et al. A randomized, multicentre, open-label phase II proof-of-concept trial investigating the clinical efficacy and safety of the addition of convalescent plasma to the standard of care in patients hospitalized with COVID-19: the Donated Antibodies Working against nCoV (DAWn-Plasma) trial. Trials 2020; 21: 981.

19 Rubin DB. Multiple imputation after 18+ years. J Am Stat Assoc 1996; 91: 473-489.

20 Abani O, Abbas A, Abbas F, et al. Convalescent plasma in patients admitted to hospital with COVID-19 (RECOVERY): a randomised controlled, open-label, platform trial. Lancet 2021; 397: 2049-2059.

21 O'Donnell MR, Grinsztejn B, Cummings MJ, et al. A randomized double-blind controlled trial of convalescent plasma in adults with severe COVID-19. J Clin Invest 2021; 131: e150646.

22 Betrains A, Godinas L, Woei-A-Jin FJSH, et al. Convalescent plasma treatment of persistent severe acute respiratory syndrome coronavirus-2 (SARS-CoV-2) infection in patients with lymphoma with impaired humoral immunity and lack of neutralising antibodies. Br J Haematol 2021; 192: 1100-1105.

23 Hueso $\mathrm{T}$, Pouderoux C, Péré $\mathrm{H}$, et al. Convalescent plasma therapy for B-cell-depleted patients with protracted COVID-19. Blood 2020; 136: 2290-2295. 